Research article

\title{
Gain-scheduled predictive extended state observer for time-varying delays systems with mismatched disturbances
}

\author{
Antonio Gonzalez ${ }^{\mathrm{a}, *}$, Vicente Balaguer ${ }^{\mathrm{b}}$, Pedro Garcia ${ }^{\mathrm{b}}$, Angel Cuenca ${ }^{\mathrm{b}}$ \\ a Instituto de Investigación en Ingeniería de Aragón, Universidad de Zaragoza, 50018, Spain \\ ${ }^{\mathrm{b}}$ Instituto de Automática e Informática Industrial, Universitat Politècnica de València, 46020, Spain
}

\section{H I G H L I G H T S}

- A novel predictor-based control with extended state observer (ESO) is given.

- Time-varying delays are considered both in input and output channels.

- A delay-dependent gain scheduling strategy is integrated with the ESO.

- The disturbance rejection and performance are improved compared with other similar approaches.

- The effectiveness of the proposed method is validated through simulation and experimental setup.

\section{A R T I C L E I N F O}

\section{Article history:}

Received 23 April 2018

Received in revised form 20 September 2018

Accepted 24 September 2018

Available online $\mathrm{xxxx}$

\section{Keywords:}

Time-varying delay

Digital control implementation

Mismatched disturbance

Predictor-based control

Gain-scheduling

Extended state observer

\begin{abstract}
A B S T R A C T
In this paper, a novel control scheme for systems with input and output time-varying delays is provided in discrete-time domain. The control strategy combines predictor-like techniques with a delay-dependent gain-scheduled extended state observer. The main goal is twofold: (i) to minimize the negative effect of time-varying delays in the closed-loop performance and, (ii) to actively compensate the effect of mismatched disturbances in the controlled output. Moreover, a sufficient condition based on Linear Matrix Inequalities (LMI) is provided to obtain the maximum delay interval that ensures the stability of the closed-loop system. Finally, the achieved benefits of the proposal are shown by simulation in open-loop unstable plants, and experimentally validated in a test-bed quadrotor platform.
\end{abstract}

(c) 2018 ISA. Published by Elsevier Ltd. All rights reserved.

\section{Introduction}

Time delays may cause performance deterioration, or even instability of the control system if they are not taken into account in the control design [1]. With the objective of counteracting the negative effects of time delays in the closed-loop control, different time delay compensation strategies have been proposed in the literature (see, e.g., [2-4] and references therein). In particular, the predictor-feedback approach uses a transformation that relates the

This work was supported in part by European Commission as part of Project H2020-SEC-2016-2017, Topic: SEC-20-BES-2016 - Id: 740736 - "C2 Advanced Multi-domain Environment and Live Observation Technologies", in part by the Spanish Ministry of Economy, Industry, and Competition under Project TIN201786520-C3-1-R, in part by Universitat Politècnica de València under Project FPIUPV 2017 PAID-01-17, and in part by Spanish Government/European Union Project DPI2015-69376-R (MINECO/FEDER).

* Corresponding author.

E-mail addresses: angonsor@unizar.es (A. Gonzalez),vibagar3@upvnet.upv.es (V. Balaguer), pggil@isa.upv.es (P. Garcia), acuenca@isa.upv.es (A. Cuenca). original delayed system with another delay-free system, simplifying thus the control design [5]. Nevertheless, the presence of timevarying delays may degrade the closed-loop behavior and brings extra difficulties in the control synthesis. In discrete-time framework, some studies carried out the closed-loop stability analysis and the design of the predictor-feedback control with time-varying input delays [6,7], and time-varying output delays [8]. However, these studies did not take into account the simultaneous presence of time-varying delays in both channels.

On the other hand, a large number of control systems are usually affected by unmeasurable external disturbances. In the aim of minimizing their negative impact in the control loop, different approaches were proposed under the so-called Disturbance Observer-Based Control (DOBC) methods [9-13]. In particular, the Extended State Observer (ESO) became more popular than other DOBC approaches because no prior knowledge of the system plant is required, except for the system relative degree [9]. In case of mismatched uncertainties, that is to say, when the disturbance does not affect the system in the same channel as the control action, the disturbance rejection problem is more difficult to handle [14, 
15]. For systems with input delays, a modified ESO was proposed in $[16,17]$ by simply considering a delayed input in the ESO scheme to improve the disturbance rejection. Other related contributions directly integrated the ESO with predictor-feedback approaches in [18]. However, these works assumed that the plant state is fully measurable, and dealt only with matched uncertainties. Although these limitations were further overcome in [19] and extended to discrete-time systems under predictor-feedback control in [2022 ], all these works were restricted to time-constant input delays. Therefore, to the best of the authors' knowledge, the synthesis of predictor-feedback control and ESO under the simultaneous presence of time-varying input and time-varying output delays, together with mismatched disturbances, has not been previously investigated, which motivates our study.

In this paper, we combine a predictor-feedback control with a properly designed gain-scheduled ESO to counteract the effect of time-varying delays in both channels. Therefore, the closedloop performance is improved with respect to other recent related works that only considers time-constant delays [20]. Moreover, the mismatched disturbances are effectively compensated in the controlled output. Furthermore, we give a sufficient condition based on LMIs, which allows to easily obtain the maximum delay intervals under which the system is stable for any time-varying delay, no matter how fast it may vary.

The remainder of the paper is structured as follows. In Section 2 , the problem statement and some preliminary results are introduced. In Section 3, the proposed control structure is presented. Section 4 presents the stability analysis for the control system. In Section 5, simulation examples are presented. In Section 6, the control solution is experimentally validated using a quadrotor platform. Finally, some conclusions and perspectives are outlined in Section 7.

\section{Problem statement and preliminaries}

Consider the following discrete-time system model:

$x_{k+1}=A x_{k}+B u_{k-d_{k}^{I}}+B_{w} w_{k}$

where $x_{k} \in \mathcal{R}^{n}$ is the system, $u_{k} \in \mathcal{R}^{m}$ is the control action subject to the input delay $d_{k}^{I}, w_{k} \in \mathcal{R}^{q}$ is a mismatched external disturbance. Also, consider the output systems:

$y_{k}=C x_{k-d_{k}^{0}}, \quad y_{s, k}=C_{s} x_{k}$,

where $y_{k} \in \mathcal{R}^{p}$ is the measured output subject to the output delay $d_{k}^{O}$, and $y_{s, k} \in \mathcal{R}^{p_{s}}$ is the controlled output.

In this paper, the following assumptions are made:

Assumption 1. The input and output delays $d_{k}^{I}, d_{k}^{O}$ are assumed to be unknown time-varying but measurable during control execution, and bounded by:

$h_{1}^{I} \leq d_{k}^{I} \leq h_{2}^{I}$,

$h_{1}^{O} \leq d_{k}^{O} \leq h_{2}^{O}$,

where each pair $\left(h_{1}^{I}, h_{2}^{I}\right)$ and $\left(h_{1}^{O}, h_{2}^{O}\right)$ are known.

Assumption 2. For some unknown initial value $w_{0}$, the disturbance signal $w_{k}$ can be modeled as:

$w_{k+1}=A_{w} w_{k}$

being $A_{w}$ a know matrix. This implies that the amplitude of the disturbance is unknown, but the type of disturbance to be rejected should be known: e.g., steps, ramps, or sinusoidals with a given frequency.
Assumption 3. There exist $K$ and $\mathcal{L}$ such that the matrices $A+B K$ and $\mathcal{A}-\mathcal{L C}$ are Schur stable, where

$\mathcal{A}=\left[\begin{array}{cc}A & B_{w} \\ 0 & A_{w}\end{array}\right], \quad \mathcal{C}=\left[\begin{array}{ll}C & 0_{p \times q}\end{array}\right]$.

Note that time-varying perturbations on delays might cause poor performance or even instability in the closed-loop control when the delay intervals are large enough. Therefore, our objective is to design a control law $u_{k}=f\left(y_{k}\right)$ with time delay compensation for system (1) such that: (i) the closed-loop is stabilized with larger delay intervals, and (ii) the steady-state error in the controlled output $y_{s, k}$ due to the disturbance $w_{k}$ is rejected, for any timevarying delays $d_{k}^{I}, d_{k}^{O}$ satisfying Assumption 1 .

The following preliminary results will be useful for further developments:

Lemma 1. Consider the following Artstein's state transformation [2]:

$z_{k}=x_{k}+\Phi_{k}\left(h_{1}^{I}\right)+\Phi_{k}\left(h_{2}^{I}\right)$

where

$\Phi_{k}\left(h_{f}^{I}\right)=\frac{1}{2} \sum_{i=0}^{h_{f}^{I}-1} A^{-i-1} B u_{k-h_{f}^{I}+i}, \quad f=1,2$

Then, system (1) can be equivalently represented by the following delay-free interconnected model:

$M_{S}:\left\{\begin{array}{l}z_{k+1}=A z_{k}+F u_{k}+\frac{\tau_{I} B}{2} w_{d, k}+B_{w} w_{k}, \\ y_{d, k}=u_{k}-u_{k-1},\end{array}\right.$

$\Delta:\left\{w_{d, k}=\Delta_{d, k} y_{d, k}, \quad\left\|\Delta_{d, k}\right\|_{\infty} \leq 1\right.$,

where $\tau_{I}=h_{2}^{I}-h_{1}^{I}$, and

$F=\left(A^{-h_{1}^{I}}+A^{-h_{2}^{I}}\right) \frac{B}{2}$

Proof. The proof can be straightforwardly outlined from [23].

Theorem 1 (Scaled Small Gain Theorem [24] Chapter 8). The interconnected system on Fig. 1 is robustly stable for any interconnected timevarying uncertain system $\Delta$ with $\|\Delta\|_{\infty} \leq 1$ if the following two conditions hold: (i) The system $M_{S}$ is internally stable and (ii) there exist regular matrices $T_{1}, T_{2}$ such that $T_{1} \Delta=\Delta T_{2}$ and $\left\|T_{2} M_{S} T_{1}^{-1}\right\|_{\infty}<1$.

\section{Gain-scheduled predictor-observer control strategy}

In this section, we first present the control strategy. Second, we demonstrate that an equivalent delay-free interconnected model can be found for the closed-loop control system formed by (1) and the proposed control law. Other key aspects, such as the advantages in terms of closed-loop performance enhancement under time-varying delays, are discussed in Section 3.3.

\subsection{Proposed control scheme}

Let us introduce the following control strategy:

$u_{k}=\mathcal{K} \hat{z}_{k}+K_{w} \hat{w}_{k}$,

where $\hat{z}_{k}$ and $\hat{w}_{k}$ are obtained from the gain-scheduled predictive ESO given below:

$\hat{\bar{z}}_{k+1}=\mathcal{A} \hat{\bar{z}}_{k}+\mathcal{F} u_{k}+\mathcal{A}^{d_{k}^{O}} \mathcal{L}\left(\bar{y}_{k}-\mathcal{C} \mathcal{A}^{-d_{k}^{O}} \hat{\bar{z}}_{k}\right)$, 
where $\hat{\bar{z}}_{k}^{T}=\left[\begin{array}{ll}\hat{z}_{k}^{T} & \hat{w}_{k}^{T}\end{array}\right]$, the matrices $\mathcal{A}$ and $\mathcal{C}$ are defined in (5), and $\mathcal{F}=\left[\begin{array}{ll}F^{T} & 0\end{array}\right]^{T}$, being $F$ defined in (8). The prediction of the output system is obtained as:

$\bar{y}_{k}=y_{k}+C A^{-d_{k}^{O}}\left(\Phi_{k}\left(h_{1}^{I}\right)+\Phi_{k}\left(h_{2}^{I}\right)+\Omega_{k}\left(d_{k}^{O}\right)\right)$,

where the operators $\Phi_{k}\left(h_{1}^{I}\right), \Phi_{k}\left(h_{2}^{I}\right)$ involved in the Artstein's reduction method are defined in (7), and $\Omega_{k}\left(d_{k}^{O}\right)$ is defined as:

$\Omega_{k}\left(d_{k}^{O}\right)=\sum_{i=0}^{d_{k}^{0}-1} A^{d_{k}^{0}-i-1} B u_{k-d_{k}^{O}+i-d_{k-d_{k}^{O}+i}^{D}}$.

The control gain $\mathcal{K}$ in (9) is defined as $\mathcal{K}=2 K\left(A^{-h_{1}^{l}}+A^{-h_{2}^{l}}\right)^{-1}$, where $K$ is determined in such a way that $A+B K$ is Schur stable (according to Assumption 3). In the same way, the observer gain $\mathcal{L}$ in (10) is defined to be $\mathcal{A}-\mathcal{L C}$ Schur stable. The parameter $K_{w}$ in (9) is tuned to compensate the steady-state error of the disturbance $w_{k}$ in the controlled output $y_{s, k}$ (more details in Remark 1, at the end of Section 3.3)

The gain-scheduled strategy is implemented through the delaydependent terms $A^{d_{k}^{0}}, A^{-d_{k}^{0}}$ in (10) and (11), together with the operator (12). Thus, the closed-loop poles are kept to be the same as the eigenvalues of $A+B K$ and $\mathcal{A}-\mathcal{L C}$ regardless of time delays, as discussed later in Section 3.3. Notice also that the conventional ESO is obtained from (10) in the absence of time delays $\left(d_{k}^{I} \equiv 0, d_{k}^{0} \equiv\right.$ $0)$.

\subsection{Delay-free closed-loop model description}

The following theorem finds a delay-free interconnected statespace model for the closed-loop system formed by (1) and the given control in (9):

Theorem 2. The closed-loop system formed by the system (1) and the control law (9) can be expressed as the interconnected system formed by the delay-free model $\mathcal{M}_{S}$ and the feedback system $\Delta$ :

$M_{S}:\left\{\begin{array}{l}\xi_{k+1}=\bar{A}_{k} \xi_{k}+\bar{G} w_{d, k}+\bar{B}_{w, k} w_{k}, \\ y_{d, k}=\bar{H} \xi_{k},\end{array}\right.$
$\Delta:\left\{\begin{array}{l}w_{d, k}=\Delta_{d, k} y_{d, k}, \quad\left\|\Delta_{d, k}\right\|_{\infty} \leq 1,\end{array}\right.$

where $\xi_{k}^{T}=\left[\begin{array}{llll}z_{k}^{T} & u_{k-1}^{T} & \hat{z}_{k}^{T} & \hat{w}_{k}^{T}\end{array}\right]$, and

$\bar{A}_{k}=\left[\begin{array}{cccc}A & 0 & F \mathcal{K} & F K_{w} \\ 0 & 0 & \mathcal{K} & K_{w} \\ \Pi_{1, k} & 0 & A-\Pi_{1, k}+F \mathcal{K} & B_{w}-\Pi_{2, k}+F K_{w} \\ \Pi_{3, k} & 0 & -\Pi_{3, k} & I_{q}-\Pi_{4, k}\end{array}\right]$,

$\bar{G}=\left[\begin{array}{l}1 \\ 0 \\ 0 \\ 0\end{array}\right] \otimes\left(\frac{\tau_{1} B}{2}\right), \quad \bar{B}_{w, k}=\left[\begin{array}{c}B_{w} \\ 0 \\ \Pi_{2, k} \\ \Pi_{4, k}\end{array}\right]$,

$\bar{H}=\left[\begin{array}{llll}0_{m \times n} & -I_{m} & \mathcal{K} & K_{w}\end{array}\right]$,

$\tau_{I}=h_{2}^{I}-h_{1}^{I}$,

being $\Pi_{i, k}, i=1,2,3,4$ the matrices obtained from the following partition:

$\mathcal{A}^{d_{k}^{o}} \mathcal{L} \mathcal{C A}^{-d_{k}^{O}}=\left[\begin{array}{ll}\Pi_{1, k} & \Pi_{2, k} \\ \Pi_{3, k} & \Pi_{4, k}\end{array}\right]$,

with $\Pi_{1, k} \in \mathcal{R}^{n}$, and the rest of matrices $\Pi_{2, k}, \Pi_{3, k}, \Pi_{4, k}$ of compatible dimensions.

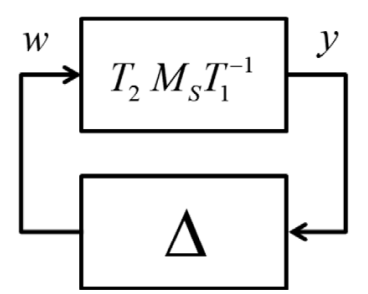

Fig. 1. Interconnected system: $M_{S}$ is a known system, and $\Delta$ is some unknown unitary norm-bounded system, which contains all sources of time-delays variations. Matrices $T_{1}, T_{2}$ are free and must satisfy $T_{1} \Delta=\Delta T_{2}$.

Proof. Following the original ideas of the ESO [9], the following state-space augmented model can be considered from (1) and (4):

$\left\{\begin{array}{l}\bar{x}_{k+1}=\mathcal{A} \bar{x}_{k}+\mathcal{B} u_{k-d_{k}^{I}} \\ y_{k}=\mathcal{C} \bar{x}_{k-d_{k}^{o}}, \quad y_{s, k}=\mathcal{C}_{s} \bar{x}_{k},\end{array}\right.$

where $\bar{x}^{T}=\left[\begin{array}{ll}x_{k}^{T} & w_{k}^{T}\end{array}\right]$, the matrices $\mathcal{A}$ and $\mathcal{C}$ are defined in (5), and

$\mathcal{B}=\left[\begin{array}{l}B \\ 0\end{array}\right], \quad \mathcal{C}_{s}=\left[\begin{array}{ll}C_{s} & 0\end{array}\right]$.

Now, define the augmented state $\bar{x}_{k}=\left[\begin{array}{ll}x_{k}^{T} & w_{k}\end{array}\right]^{T}$. From (15), we have that the exact $h$-step ahead state prediction can be obtained as:

$\bar{x}_{k+h}=\mathcal{A}^{h} \bar{x}_{k}+\bar{\Omega}_{k+h}(h)$,

where $\bar{\Omega}_{k+h}(h)=\left[\begin{array}{ll}\Omega_{k+h}^{T}(h) & 0_{1 \times q}\end{array}\right]^{T}$ and $\Omega_{k}(h)$ is the operator defined in (12). The $h$-step back of the above expression yields:

$\bar{x}_{k}=\mathcal{A}^{h} \bar{x}_{k-h}+\bar{\Omega}_{k}(h)$.

Multiplying both-sides of (18) by $\mathcal{C} \mathcal{A}^{-h}$ we obtain:

$\mathcal{C} \mathcal{A}^{-h} \bar{x}_{k}=\mathcal{C} \bar{x}_{k-h}+\mathcal{C} \mathcal{A}^{-h} \bar{\Omega}_{k}(h)$.

which is equivalent to:

$\mathcal{C} \mathcal{A}^{-h} \bar{x}_{k}=C x_{k-h}+\mathcal{C} \mathcal{A}^{-h} \bar{\Omega}_{k}(h)$.

Replacing $h$ by $d_{k}^{o}$ we have:

$\mathcal{C} \mathcal{A}^{-d_{k}^{0} \bar{x}_{k}}=\underbrace{C x_{k-d_{k}^{0}}}_{y_{k}}+\mathcal{C} \mathcal{A}^{-d_{k}^{0}} \bar{\Omega}_{k}\left(d_{k}^{0}\right)$,

which leads to:

$y_{k}=\mathcal{C} \mathcal{A}^{-d_{k}^{0}} \bar{x}_{k}-\mathcal{C} \mathcal{A}^{-d_{k}^{0}} \bar{\Omega}_{k}\left(d_{k}^{0}\right)$.

Now, replacing $\bar{y}_{k}$ defined in (11) into (10) we have:

$\hat{\bar{z}}_{k+1}=\mathcal{A} \hat{\bar{z}}_{k}+\mathcal{F} u_{k}+\mathcal{A}^{d_{k}^{0}} \mathcal{L} y_{k}$

$+\mathcal{A}_{k}^{d_{k}^{0}} \mathcal{L} C A^{-d_{k}^{0}}\left(\Phi_{k}\left(h_{1}^{I}\right)+\Phi_{k}\left(h_{2}^{I}\right)+\Omega_{k}\left(d_{k}^{0}\right)\right)$

$-\mathcal{A}^{d_{k}^{0}} \mathcal{L} \mathcal{C} \mathcal{A}^{-d_{k}^{0}} \hat{\bar{z}}_{k}$.

Substituting $y_{k}$ from (22) into the above expression (23), and taking into account that $\mathcal{C} \mathcal{A}^{-d_{k}^{O}} \bar{\Omega}_{k}\left(d_{k}^{O}\right)=C A^{-d_{k}^{O}} \Omega_{k}\left(d_{k}^{O}\right)$, we have:

$\hat{\bar{z}}_{k+1}=\mathcal{A} \hat{\bar{z}}_{k}+\mathcal{F} u_{k}$

$+\mathcal{A}^{d_{k}^{0}} \mathcal{L} C A^{-d_{k}^{0}}\left(\Phi_{k}\left(h_{1}^{I}\right)+\Phi_{k}\left(h_{2}^{I}\right)\right)$

$+\mathcal{A}^{d_{k}^{0}} \mathcal{L} \mathcal{C} \mathcal{A}^{-d_{k}^{0}}\left(\bar{x}_{k}-\hat{\bar{z}}_{k}\right)$.

Note from the definition of $\bar{x}_{k}$ and $\bar{\Phi}_{k}(h)$ that the equivalence $x_{k}=$ $z_{k}-\Phi_{k}\left(h_{1}^{I}\right)-\Phi_{k}\left(h_{2}^{I}\right)$ deduced from (6) implies $\bar{x}_{k}=\bar{z}_{k}-\bar{\Phi}_{k}\left(h_{1}^{I}\right)-$ $\bar{\Phi}_{k}\left(h_{2}^{I}\right)$, where $\bar{\Phi}_{k}\left(h_{i}^{I}\right)=\left[\begin{array}{ll}\Phi_{k}^{T}\left(h_{i}^{I}\right) & 0_{1 \times q}\end{array}\right]^{T}, i=1,2$ and $\bar{z}_{k}=$ 
$\left[\begin{array}{ll}z_{k}^{T} & w_{k}^{T}\end{array}\right]^{T}$. Then, replacing $\bar{x}_{k}$ into (24), and taking into account that $\mathcal{C} \mathcal{A}^{-d_{k}^{0}} \bar{\Phi}_{k}\left(h_{i}^{I}\right)=C A^{-d_{k}^{0}} \Phi_{k}\left(h_{i}^{I}\right), i=1,2$, we obtain:

$\hat{\bar{z}}_{k+1}=\mathcal{A} \hat{\bar{z}}_{k}+\mathcal{F} u_{k}+\mathcal{A}^{d_{k}^{0}} \mathcal{L} \mathcal{C} \mathcal{A}^{-d_{k}^{0}}\left(\bar{z}_{k}-\hat{\bar{z}}_{k}\right)$.

Consider the matrix partition defined in (14). Then, (25) is equivalent to:

$$
\left\{\begin{aligned}
\hat{z}_{k+1} & =A \hat{z}_{k}+F u_{k}+\Pi_{1, k}\left(z_{k}-\hat{z}_{k}\right) \\
& +\left(B_{w}-\Pi_{2, k}\right) \hat{w}_{k}+\Pi_{2, k} w_{k} \\
\hat{w}_{k+1} & =\left(I_{q}-\Pi_{4, k}\right) \hat{w}_{k}+\Pi_{3, k}\left(z_{k}-\hat{z}_{k}\right)+\Pi_{4, k} w_{k}
\end{aligned}\right.
$$

On the other hand, by virtue of Lemma 1 , system (1) can be put as the interconnected model between:

$$
\begin{aligned}
& {\left[\begin{array}{c}
z_{k+1} \\
u_{k}
\end{array}\right]=\left[\begin{array}{cc}
A & 0 \\
0 & 0
\end{array}\right]\left[\begin{array}{c}
z_{k} \\
u_{k-1}
\end{array}\right]+\left[\begin{array}{c}
F \\
I_{m}
\end{array}\right] u_{k}+\left[\begin{array}{c}
\tau_{I} B \\
2 \\
0
\end{array}\right] w_{d, k}} \\
& +\left[\begin{array}{c}
B_{w} \\
0
\end{array}\right] w_{k}, \\
& y_{d, k}=u_{k}-u_{k-1}
\end{aligned}
$$

Therefore, the augmented state-space representation of (26) and (27) yields:

$$
\begin{aligned}
& {\left[\begin{array}{c}
z_{k+1} \\
u_{k} \\
\hat{z}_{k+1} \\
\hat{w}_{k+1}
\end{array}\right]=\left[\begin{array}{cccc}
A & 0 & 0 & 0 \\
0 & 0 & 0 & 0 \\
\Pi_{1, k} & 0 & A-\Pi_{1, k} & B_{w}-\Pi_{2, k} \\
\Pi_{3, k} & 0 & -\Pi_{3, k} & I_{q}-\Pi_{4, k}
\end{array}\right]\left[\begin{array}{c}
z_{k} \\
u_{k-1} \\
\hat{z}_{k} \\
\hat{w}_{k}
\end{array}\right]} \\
& +\left[\begin{array}{c}
F \\
I_{m} \\
F \\
0
\end{array}\right] u_{k}+\left[\begin{array}{c}
\frac{\tau_{I} B}{2} \\
0 \\
0 \\
0
\end{array}\right] w_{d, k}+\left[\begin{array}{c}
B_{w} \\
0 \\
\Pi_{2, k} \\
\Pi_{4, k}
\end{array}\right] w_{k} .
\end{aligned}
$$

Finally, replacing $u_{k}$ from (9) into the above expression (28) and $y_{d, k}$ we obtain the interconnected system (13), concluding the proof.

\subsection{Discussion}

Notice that the input and output delays cannot be gathered into a single round-trip delay in the closed-loop system due to their time-varying nature. Therefore, they must be treated separately for compensation purposes: the input delay is compensated through the Artstein's reduction method using Lemma 1, leading to the equivalence between (1) and (27). The output delay is compensated by the gain-scheduled ESO (10) together with the output prediction defined in (11). Therefore, as demonstrated in the corollary given below, the eigenvalues of the closed-loop matrix $\bar{A}_{k}$ in (13) are those of the matrices $A+B K$ and $\mathcal{A}-\mathcal{L C}$, regardless of the time-varying delays.

Corollary 1. The eigenvalues of the matrix $\bar{A}_{k}$ in (13) are the same as $A+B K$ and $\mathcal{A}-\mathcal{L C}$, plus $m$ eigenvalues at 0 , for any instant value of time-varying delays.

Proof. Consider the following regular matrix:

$\mathcal{T}=\left[\begin{array}{cccc}I_{n} & 0 & 0 & 0 \\ 0 & I_{m} & 0 & 0 \\ -I_{n} & 0 & I_{n} & 0 \\ 0 & 0 & 0 & I_{q}\end{array}\right]$

Pre- and post multiplying $\bar{A}_{k}$ by $\mathcal{T}$ and its inverse $\mathcal{T}^{-1}$, we obtain:

$\tilde{\bar{A}}_{k}=\mathcal{T} \bar{A}_{k} \mathcal{T}^{-1}$

$$
=\left[\begin{array}{cccc}
A+F \mathcal{K} & 0 & F \mathcal{K} & F K_{w} \\
\mathcal{K} & 0 & \mathcal{K} & K_{w} \\
0 & 0 & A-\Pi_{1, k}+F \mathcal{K} & B_{w}-\Pi_{2, k}+F K_{w} \\
0 & 0 & -\Pi_{3, k} & I_{q}-\Pi_{4, k}
\end{array}\right]
$$

Note that $\tilde{\bar{A}}_{k}$ can also be written as:

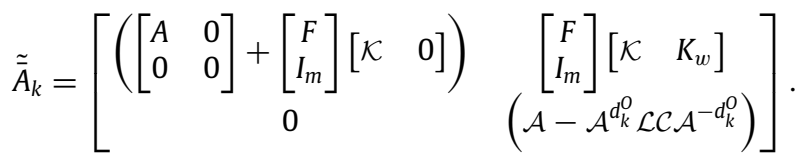

Therefore, from the block-triangular form of $\tilde{\bar{A}}_{k}$, it can be deduced that its eigenvalues are those of the matrices:

$$
\left(\left[\begin{array}{cc}
A & 0 \\
0 & 0
\end{array}\right]+\left[\begin{array}{c}
F \\
I_{m}
\end{array}\right]\left[\begin{array}{ll}
\mathcal{K} & 0
\end{array}\right]\right) \text { and }\left(\mathcal{A}-\mathcal{A}^{d_{k}^{o}} \mathcal{L C} \mathcal{A}^{-d_{k}^{0}}\right)
$$

Finally, the proof can be completed from the fact that:

- (i) The eigenvalues of $\tilde{\bar{A}}_{k}$ and $\bar{A}_{k}$ are the same.

- (ii) The eigenvalues of: $\left(\left[\begin{array}{cc}A & 0 \\ 0 & 0\end{array}\right]+\left[\begin{array}{c}F \\ I_{m}\end{array}\right]\left[\begin{array}{ll}\mathcal{K} & 0\end{array}\right]\right)$ and $\left(\mathcal{A}-\mathcal{A}^{d_{k}^{0}} \mathcal{L} \mathcal{C} \mathcal{A}^{-d_{k}^{0}}\right)$ are the same as $(A+B K)$ (plus $m$ eigenvalues at 0$)$ and $(\mathcal{A}-\mathcal{L C})$ respectively, for any value of $d_{k}^{O}$.

Remark 1. In light of Corollary 1 , it can be deduced that the closed-loop poles of system (13) are not affected by $K_{w}$. Therefore, provided that the dimensions of $w_{k}$ and $y_{s, k}$ are the same, or the generalized rank conditions [14] hold, the parameter $K_{w}$ can be designed to cancel the steady-state error due to the disturbance $w_{k}$ on the controlled output $y_{s, k}$. The proper value of $K_{w}$ can be deduced from the Final Value Theorem by considering the augmented system state-space model of system (26) with $\left[x_{k}^{T}, u_{k-1}^{T}, \cdots, u_{k-h_{2}^{I}}^{T}, \hat{x}_{k}^{T}, \hat{w}_{k}^{T}\right]^{T}$, and the transfer function from $w_{k}$ to the controlled output $y_{s, k}$. In case of $w_{k}$ is in step form, we have that $K_{w}=-\bar{\Xi}_{1}^{-1} \bar{\Xi}_{2}$, where:

$$
\begin{aligned}
& \bar{\Xi}_{1}=\left(\bar{C}_{s}(I-\bar{A}-\bar{F} \bar{K})^{-1} \bar{F}\right), \\
& \bar{\Xi}_{2}=\left(\bar{C}_{s}(I-\bar{A}-\bar{F} \bar{K})^{-1} \bar{B}_{w}\right),
\end{aligned}
$$

and

$\bar{C}_{s}=\left[\begin{array}{llllll}C_{s} & \gamma_{1} C_{s} A^{-1} B & \cdots & \gamma_{h_{2}^{I}} C_{s} A^{-h_{2}^{I} B} & 0 & 0\end{array}\right]$,

$\gamma_{i}, i=1, \ldots, h_{2}^{I}= \begin{cases}1 & \text { if } i \leq h_{1}^{I} \\ 0 & \text { otherwise, }\end{cases}$

$\bar{A}=\left[\begin{array}{ccc}A & 0 & 0 \\ 0 & 0_{m \times(m-1) \cdot h_{2}^{I}} & 0_{m \times m} \\ 0 & I_{(m-1) \cdot h_{2}^{I}} & 0\end{array}\right]$,

$\bar{F}^{T}=\left[\begin{array}{lll}F^{T} & I_{m} & 0_{m \times m\left(h_{2}^{I}-1\right)}\end{array}\right]$,

$\bar{K}=\left[\begin{array}{lll}\mathcal{K} & 0 & 0_{m \times m\left(h_{2}^{I}-1\right)}\end{array}\right]$,

$\bar{B}_{w}^{T}=\left[\begin{array}{ll}B_{w}^{T} & 0_{q \times m\left(h_{2}^{I}\right)}\end{array}\right]$.

\section{Stability analysis}

The following theorem allows to prove the stability with decayrate $0<\beta \leq 1$ of the closed-loop system (1) with the control law (9) and the predictor-observer scheme (10), for any arbitrary fasttime varying delays $d_{k}^{O}, d_{k}^{I}$ : 
Theorem 3. Given some control and observer gains $\mathcal{K}$, and $\mathcal{L}$, the closed-loop system (1) with the control law (9) and the predictorobserver scheme (10) is robustly asymptotically stable with decay rate $\beta$ if there exists a symmetric matrix $P \in \mathcal{R}^{2 n+m+q}>0$ such that the following LMIs are satisfied, $\forall i=1, \ldots, h_{2}^{O}-h_{1}^{O}+1$ :

$\hat{\Gamma}_{i}<0$,

where

$\hat{\Gamma}_{i}=\left[\begin{array}{cccc}-\beta^{2} P & 0 & \hat{\bar{A}}_{i}^{T} P & \bar{H}^{T} \\ (*) & -I & \bar{G}^{T} P & 0 \\ (*) & (*) & -P & 0 \\ (*) & (*) & (*) & -I\end{array}\right]$,

and

$\hat{\bar{A}}_{i}=\left[\begin{array}{cccc}A & 0 & F \mathcal{K} & F K_{w} \\ 0 & 0 & \mathcal{K} & K_{w} \\ \Pi_{1, i} & 0 & A-\Pi_{1, i}+F \mathcal{K} & B_{w}-\Pi_{2, i}+F K_{w} \\ \Pi_{3, i} & 0 & -\Pi_{3, i} & I_{q}-\Pi_{4, i}\end{array}\right]$,

where

$\mathcal{A}^{d_{i}} \mathcal{L C} \mathcal{A}^{-d_{i}}=\left[\begin{array}{ll}\Pi_{1, i} & \Pi_{2, i} \\ \Pi_{3, i} & \Pi_{4, i}\end{array}\right]$,

being $d_{i}=h_{1}^{O}+i-1$ and matrices $\bar{G}, \bar{H}$ defined in (13).

Proof. Consider the Lyapunov function $V_{k}=\xi_{k}^{T} P \xi_{k}$, where $P>0$ and $\xi_{k}^{T}$ defined in (13). The system $M_{S}$ in (13) is asymptotically stable with decay rate $\beta$, say $\left\|\xi_{k}\right\| \leq \Omega\left\|\xi_{0}\right\|^{-\beta}, \forall k \geq 0$, for some arbitrary $\Omega>0$ and any initial condition $\xi_{0}$, if the following condition holds:

$\Delta_{\beta} V_{k}=V_{k+1}-\beta^{2} V_{k}<0$.

On the other hand, it is well-known that the following condition along $\left(M_{S}\right)$ :

$\Delta_{\beta} V_{k}+y_{d, k}^{T} y_{d, k}-w_{d, k}^{T} w_{d, k}$,

$<\epsilon\left(\left\|\xi_{k}\right\|^{2}+\left\|\bar{w}_{d, k}\right\|^{2}\right) \leq 0$,

for some $\epsilon>0$, guarantees that the $H_{\infty}$ norm of system $T_{2} M_{S} T_{1}^{-1}$ is less than 1 , where $T_{1}=T_{2}=I_{m}$. From (13), the expression (38) yields:

$\xi_{k}^{T}\left(\bar{A}_{k}^{T} P \bar{A}_{k}-\beta^{2} P+\bar{H}^{T} \bar{H}\right) \xi_{k}+\xi_{k}^{T} \bar{A}_{k}^{T} P \bar{G} w_{d, k}$

$+w_{d, k}^{T} \bar{G}^{T} P \bar{A}_{k} \xi_{k}+w_{d, k}^{T}\left(\bar{G}^{T} P \bar{G}-I_{m}\right) w_{d, k}<0$

Note that $\bar{A}_{k}=\sum_{i=1}^{r} \mu_{i}\left(d_{k}^{O}\right) \hat{\bar{A}}_{i}$, where $r=h_{2}^{O}-h_{1}^{O}+1, \hat{\bar{A}}_{i}$ is defined in (35), and

$\mu_{i}\left(d_{k}^{O}\right)= \begin{cases}1 & \text { if } d_{k}^{O}-h_{1}^{O}+1=i \\ 0 & \text { otherwise }\end{cases}$

Applying Schur Complement and the above expression for $\bar{A}_{k}$, the inequality (39) is equivalent to:

$\sum_{i=1}^{r} \mu_{i}\left(d_{k}^{O}\right) \hat{\Gamma}_{i}<0$,

where $\hat{\Gamma}_{i}$ is defined in (34). Taking into account that the functions $\mu_{i}($.$) in (40) satisfy the convex sum properties: \sum_{i=1}^{r} \mu_{i}()=$. $1,0 \leq \mu_{i}() \leq$.1 , a sufficient condition for (41) is given in (33).

\section{Simulation results}

Two examples are provided in this section. Example 1 gives simulation results to show the effectiveness of the proposed control
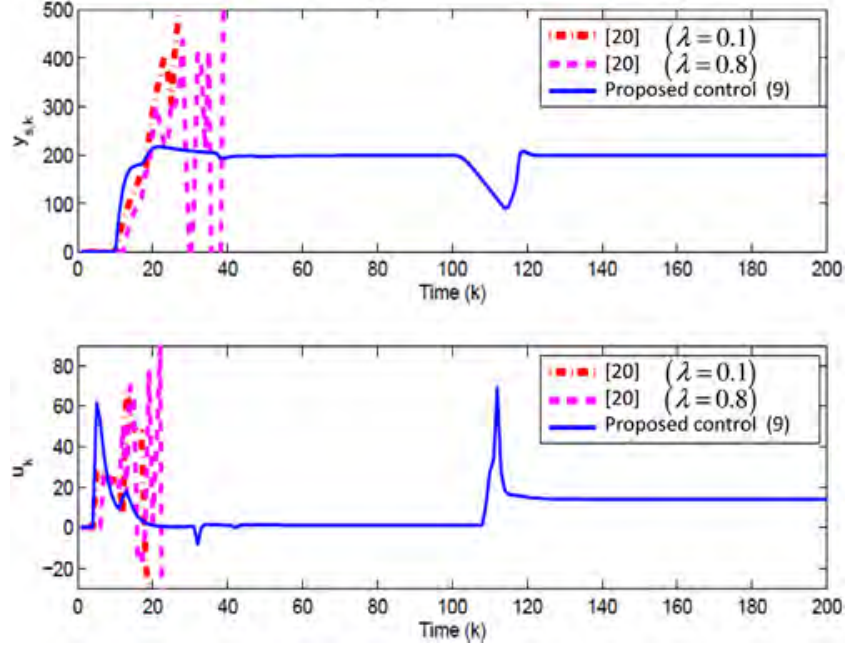

Fig. 2. Comparative results (Example 1) between two control settings given in [20] (dashed and dash-dotted lines) and the proposed control (solid line) for $d_{k}^{I}=5$ and $1 \leq d_{k}^{O} \leq 6$. Output system $y_{s, k}$ (upper-side) and control action $u_{k}$ (lower-side).

strategy by comparison with other similar approaches published in literature therein, and Example 2 simulates the closed-loop response of an open-loop unstable system, which corresponds to the experimental platform of Section 6.

\subsection{Example 1}

Let us consider the following example, already studied in [20]. The plant consists in an injection molding process with input and output delays subject to load disturbance. The discrete-time system model is (1) with system matrices:

$A=\left[\begin{array}{cc}1.607 & 1 \\ -0.6086 & 0\end{array}\right], B=\left[\begin{array}{c}1.2390 \\ -0.9282\end{array}\right], B_{w}=\left[\begin{array}{l}1 \\ 1\end{array}\right]$

$C=\left[\begin{array}{ll}1 & 1\end{array}\right], C_{s}=\left[\begin{array}{ll}1 & 0\end{array}\right]$

The simulation results given in Fig. 2 compare the time evolution of the output system $y_{s, k}$ (upper-side) and the control action $u_{k}$ (lower-side) under the two different control settings given in Example 1 [20] (dash-dotted and dashed lines respectively) with our proposed control scheme (9) (solid-line). A time-varying output delay $1 \leq d_{k}^{O} \leq 6$ has been assumed, together with an input delay $d_{k}^{I}=5$. Fig. 3 depicts the same comparative results as Fig. 2, but assuming an input delay $d_{k}^{I}=15$.

A time-constant disturbance $w_{k}$ in the form (4) with $A_{w}=I_{q}$ has been assumed in all the performed simulations, corresponding to the following step signal:

$w_{k}= \begin{cases}0 & 0 \leq k \leq 100 \\ -2 & k>100\end{cases}$

For a fair comparison, the control and observer gains have been chosen to be the same as in [20]:

$K=\left[\begin{array}{ll}-1.297 & -0.8071\end{array}\right]$,

$\mathcal{L}^{T}=\left[\begin{array}{lll}1.307 & -0.4461 & 0.0875\end{array}\right]$.

Moreover, the same time-varying output delay pattern for $d_{k}^{0}$ has been used in all the performed simulations.

\subsection{Example 2}

Consider the system model (1) (which is a discrete-time approximate model of the experimental platform used afterwards for 

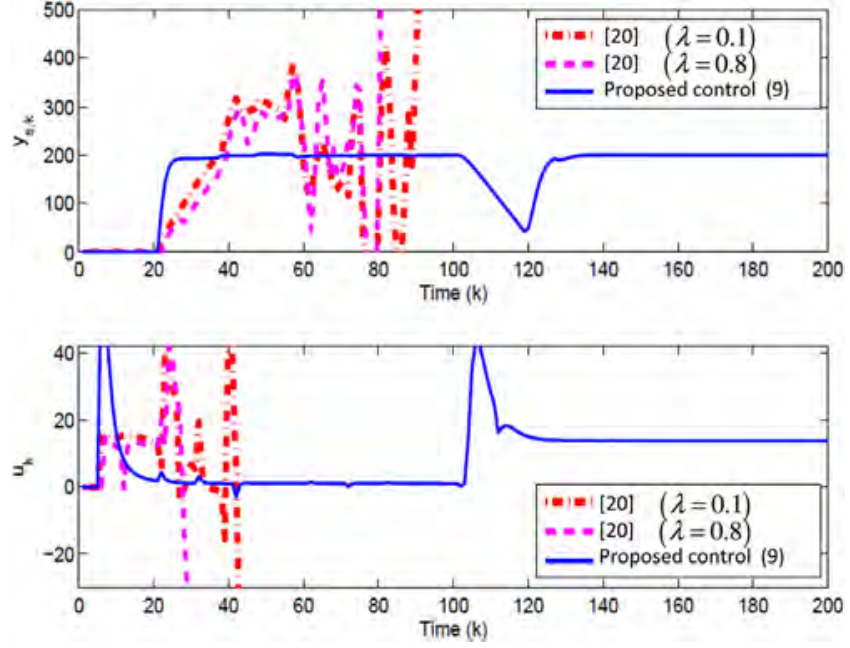

Fig. 3. Comparative results (Example 1) between two control settings given in [20] (dashed and dash-dotted lines) and the proposed control (solid line) for $d_{k}^{l}=15$ and $1 \leq d_{k}^{O} \leq 6$. Output system $y_{s, k}$ (upper-side) and control action $u_{k}$ (lower-side).

a given sampling period $T_{s}$ ), where the system matrices are given below:

$A=\left[\begin{array}{cc}1 & T_{s} \\ 0 & 1\end{array}\right], B=B_{w}=\left[\begin{array}{c}0 \\ 0.1 T_{s}\end{array}\right]$,

$C=C_{s}=\left[\begin{array}{ll}1 & 0\end{array}\right]$.

Let $T_{S}=0.01 \mathrm{~s}$. The proposed control and observer gains $K$ and $\mathcal{L}$, used to perform the simulation, are chosen such that the matrices $A+B K$ and $\mathcal{A}-\mathcal{L C}$ are Schur stable, with their respective eigenvalues $\{0.98,0.975\}$ and $\{0.50,0.96,0.97\}$ :

$K=-\left[\begin{array}{ll}50 & 45\end{array}\right], \quad \mathcal{L}^{T}=\left[\begin{array}{lll}0.57 & 3.62 & 60\end{array}\right]$,

Simulation results (controlled output $y_{s, k}$ and control action $u_{k}$ ) are depicted in Fig. 4. The controlled output corresponds to the pitch angle in degrees, and tracks a reference consisting of a sequence of steps with an amplitude of $5^{\circ}$, also depicted in Fig. 4. Moreover, it is assumed a step disturbance $w_{k}$ with amplitude -2.5 $\mathrm{V}$, introduced at $t=15 \mathrm{~s}$ The first simulation (dash-dotted line) has been performed using a conventional state-feedback control with a ESO without delays (nominal case). The second simulation (dashed line) implements the same control law as the first simulation, but assuming a randomly generated time-varying input and output delays: $1 \leq d_{k}^{O} \leq 17$ and $1 \leq d_{k}^{I} \leq 17$. The third simulation (solid-line) implements the proposed control strategy (9) with the same time-varying delay pattern as the previous simulation, for a fair comparison.

In order to check the closed-loop performance of the proposed control scheme for greater sampling periods, the simulations have also been performed assuming $T_{s}=0.05 \mathrm{~s}$ and $T_{s}=0.1 \mathrm{~s}$ with the following input and output delay intervals: $1 \leq d_{k}^{I} \leq 3,1 \leq d_{k}^{O} \leq 4$ for $T_{s}=0.05 \mathrm{~s}$, and $1 \leq d_{k}^{I} \leq 1,1 \leq d_{k}^{O} \leq 2$ for $T_{s}=0.1 \mathrm{~s}$. The obtained results have been compared in Fig. 5 with the case $T_{s}=0.01 \mathrm{~s}$ and delay intervals $1 \leq d_{k}^{I} \leq 17,1 \leq d_{k}^{O} \leq 17$.

\subsection{Discussion of the results}

In light of Figs. 2 and 3 (Example 1), it can be seen that the system becomes unstable with the two control settings given in [20] (dashed and dash-dotted line, respectively). Indeed, the closedloop instability is due to time variations in the output delay $d_{k}^{O}$, which are not considered in [20]. However, the closed-loop control
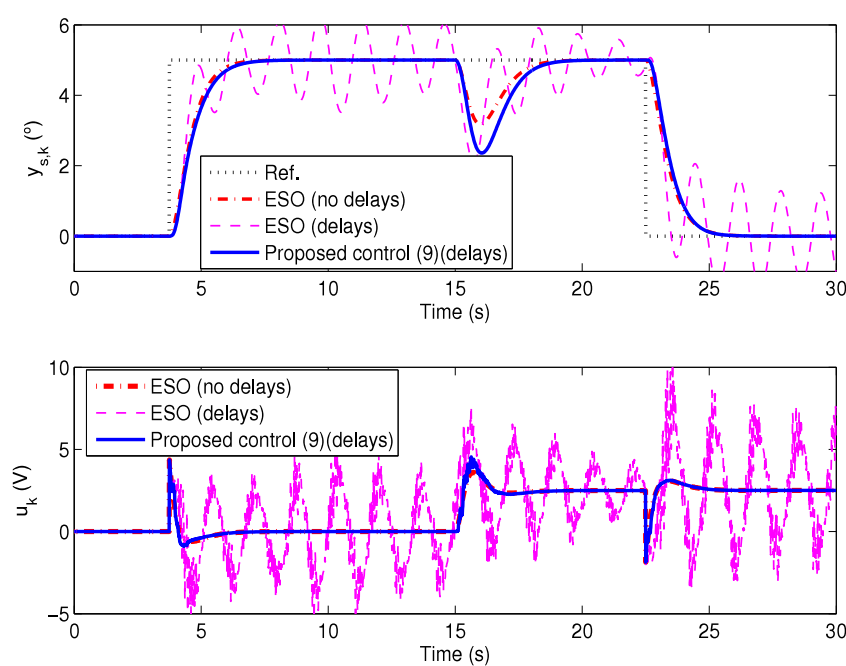

Fig. 4. Simulation results (Example 2). Dash-dotted line: state-feedback with ESO (nominal response with no delays), dashed line: state-feedback with ESO and timevarying delays : $1 \leq d_{k}^{O} \leq 17$ and $1 \leq d_{k}^{I} \leq 17$, and solid-line: proposed control scheme (9) with the same time-varying delay pattern.
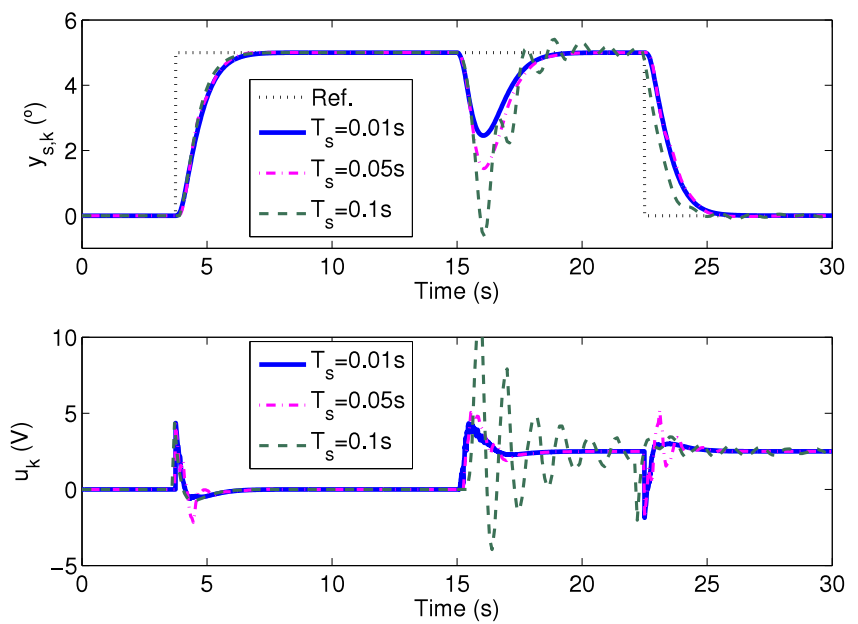

Fig. 5. Comparative results (Example 2) using the proposed control strategy for different sampling periods.

system is stabilized with the proposed control strategy (solid line) because such variations are properly counteracted by the implemented gain-scheduled approach. Furthermore, the steady-state error in the controlled output $y_{s, k}$ coming from the disturbance $w_{k}$ in (43) is canceled, despite the presence of time-varying delays (upper side of Figs. 2 and 3).

On the other hand, Theorem 3 confirms that the closed-loop system is guaranteed to be stable for any arbitrarily fast timevarying output delays $1 \leq d_{k}^{O} \leq 3$ for $d_{k}^{I}=5$, and $1 \leq d_{k}^{O} \leq 2$ for $d_{k}^{I}=15$, respectively. Notice that the maximum time delay intervals obtained by Theorem 3 are smaller than the obtained by simulation in both cases. This fact reveals that maximum allowable delay intervals obtained by applying Theorem 3 are more conservative, since the LMIs (33) are only sufficient conditions, that is to say, the most unfavorable time-varying delay pattern (worst-case) is taken into account in our analysis.

In Example 2, both time-varying delay intervals $\left(1 \leq d_{k}^{I} \leq 17\right.$, $1 \leq d_{k}^{O} \leq 17$ ) have been chosen to force the closed-loop system to the limit of stability using a conventional state-feedback control with ESO considering a sampling period $T_{s}=0.01 \mathrm{~s}$. Indeed, it can be seen in Fig. 4 that the system is almost unstable (dashed line). 


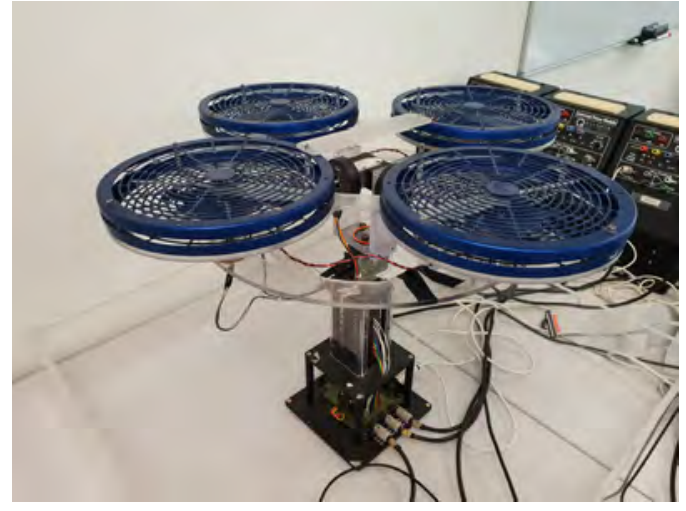

Fig. 6. 3-DOF Hover of Quanser (experimental platform).

Nevertheless, by comparing the nominal delay-free time-response (dash-dotted line) to the obtained one with the proposed control strategy under the same time-varying delays $d_{k}^{I}, d_{k}^{O}$ (solid line), it can be seen that closed-loop performance is almost recovered. On the other hand, it is proved by Theorem 3 that the stability of the closed-loop control system is guaranteed for any arbitrarily fast time-varying delay satisfying $1 \leq d_{k}^{I} \leq 5$ and $1 \leq d_{k}^{O} \leq 7$ respectively. Notice again that the maximum time delay intervals obtained by Theorem 3 are smaller than the ones obtained by simulation, such as expected from the same arguments already given in Example 1. Moreover, the proposed control strategy is shown to stabilize the system for greater sampling periods in Fig. 5. Nevertheless, it can be appreciated how the closed-loop response degrades as long as $T_{s}$ is higher, as it could be expected from the fact that the open-loop system is unstable.

\section{Experimental results}

The proposed control strategy has been implemented in the 3-DOF Hover of Quanser (see Fig. 6). It consists of a quadrotor mounted on a 3-DOF pivot joint so that the body can freely rotate in roll, pitch, and yaw angles. Our control strategy has been implemented and executed using a computer running Linux with a soft real-time patched kernel, which allows to run the full algorithm with a sampling time $T_{s}=0.01 \mathrm{~s}$. The computer is connected to the Quanser hardware by means of a data acquisition board. The angular positions are measured by optical encoders with an accuracy of $0.04^{\circ}$, and the control inputs of the system are the voltages applied to the four motors, which have an input range of $\pm 10 \mathrm{~V}$, and present a dead zone between $\pm 0.5 \mathrm{~V}$.

The controlled variable represented in the experiments is the pitch angle, which is denoted as $\theta(t)$. Assuming the yaw and roll angles are zero, the dynamics of $\theta(t)$ can be approximated by [25]:

$\ddot{\theta}(t)=K_{s} u(t)+w(t), \quad K_{s}=0.1$,

where $u(t)$ is the input voltage of the propeller, which is used to control the pitch axis torque, $K_{\mathrm{s}}=0.1$ is a constant representing the inertia moment, and $w(t)$ represents a load disturbance.

The discrete-time system model with sampling period $T_{s}=$ $0.01 \mathrm{~s}$ is the same as Example 2, with system matrices given in (45). Moreover, the control and observer gains are given in (46).

In order to experimentally validate the proposed-control law (9), three simulation have been carried out: (i) the nominal (delayfree) case with the ESO-based control, (ii) the ESO-based control with time-varying delays: $1 \leq d_{k}^{O} \leq 11$, and $1 \leq d_{k}^{I} \leq 10$, and (iii) the proposed control strategy (9) with the same time-varying delays as in case (ii).
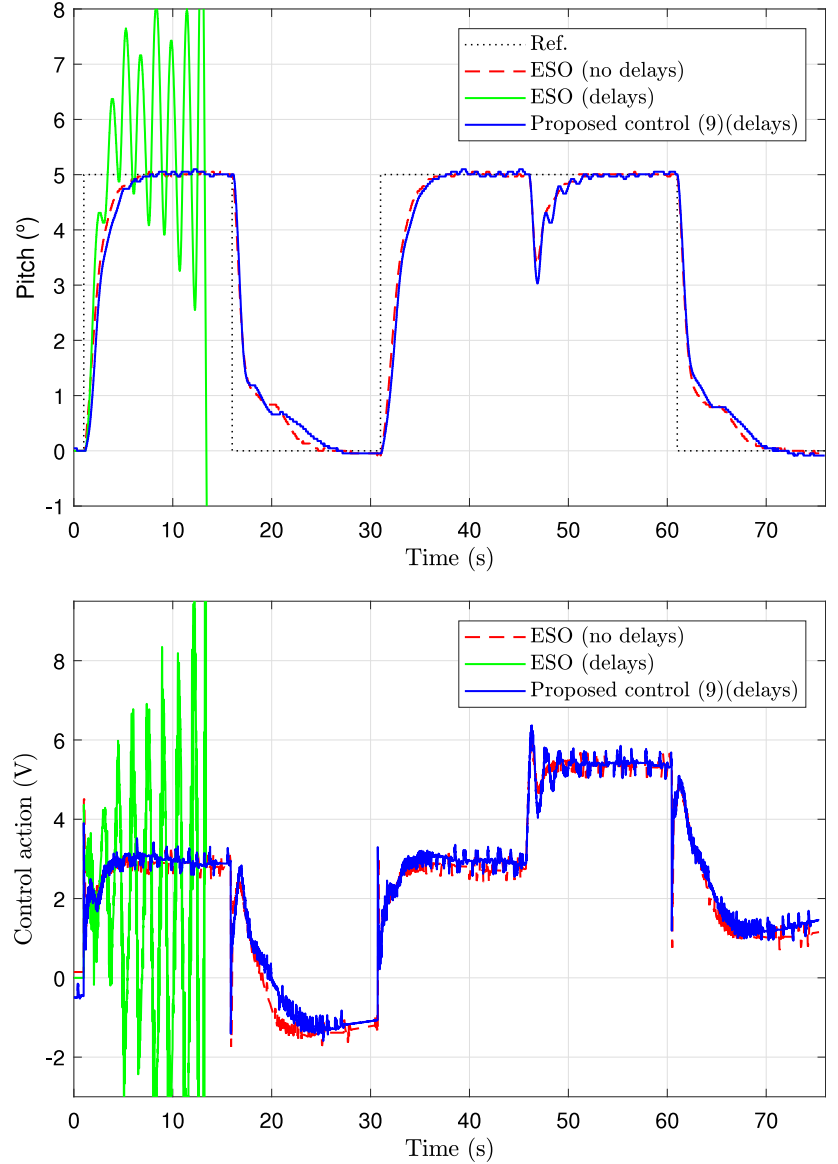

Fig. 7. Experimental results. Pitch response and the control action. A video clip of this experiment is available as complementary material.

For a fair comparison between cases (ii) and (iii), the timevarying delay patterns have been induced by software in both channels using a fixed seed of the random number generator for the repeatability of the experiments. In addition, the delay intervals have been intentionally chosen to lead the closed-loop system with the conventional ESO-based control to instability (case (ii)). The experiments have been carried out for changes of the setpoint of $5^{\circ}$, and a time-constant disturbance of $w(t)=-2.5 \mathrm{~V}$ is introduced in $t=47 \mathrm{~s}$. The response of the experiments is shown in Fig. 7, in which the improvement of our proposed strategy can be clearly appreciated: whereas the ESO-based control strategy is unstable, the proposed algorithm achieves a response very similar to the delay-free case.

\section{Conclusions and perspectives}

In this paper, a novel predictor-feedback control scheme with a delay-dependent gain-scheduled ESO has been proposed for systems with mismatched disturbances and time-varying delays in the input and output channels. Differently to other similar techniques, the proposed control scheme is able to counteract variations in time delays by means of gain-scheduled strategies, while actively compensating the steady-state error due to mismatched disturbances in the controlled output. As a result, the closed-loop poles are kept as the nominal (delay-free) behavior, improving the closed-loop performance under time-varying delays. Moreover, a sufficient LMI-based condition has been given to determine the closed-loop stability for any arbitrarily fast time-varying delays bounded by given time delay intervals. 
The effectiveness of the proposed control strategy has been illustrated through comparative simulation results and tested in open-loop unstable plants. Furthermore, it has been validated in an experimental real-time test-bed consisting in a quadrotor platform. However, the applicability of the proposed control scheme is limited to the case of unknown but measurable time-varying delays. Therefore, an appealing extension of this work could be to analyze the robustness of the proposed control scheme against uncertainties on time delay measurements.

\section{Appendix A. Supplementary data}

Supplementary material related to this article can be found online at https://doi.org/10.1016/j.isatra.2018.09.024.

\section{References}

[1] Fridman E. Introduction to time-delay systems: Analysis and control. Springer; 2014.

[2] Artstein Z. Linear systems with delayed controls: a reduction. IEEE Trans Autom Control 1982;27(4):869-79.

[3] Normey-Rico J, Camacho E. Control of dead-time processes. Springer Science \& Business Media; 2007

[4] Sanz R, Garcia P, Albertos P. A generalized smith predictor for unstable timedelay SISO systems. ISA Trans 2017;72:197-204.

[5] Lozano R, Castillo P, Garcia P, Dzul A. Robust prediction-based control for unstable delay systems: Application to the yaw control of a mini-helicopter. Automatica 2004;40(4):603-12

[6] González A, Sala A, Sanchis R. LK stability analysis of predictor-based controllers for discrete-time systems with time-varying actuator delay. Systems Control Lett 2013;62(9):764-9.

[7] Karafyllis I, Krstic M. Robust predictor feedback for discrete-time systems with input delays. Internat J Control 2013;86(9):1652-63.

[8] González A, García P, Albertos P, Castillo P, Lozano R. Robustness of a discretetime predictor-based controller for time-varying measurement delay. Control Eng Pract 2012;20(2):102-10.

[9] Han J. From PID to active disturbance rejection control. IEEE Trans Ind Electron 2009;56(3):900-6.
[10] Yang J, Zolotas A, Chen WH, Michail K, Li S. Robust control of nonlinear MAGLEV suspension system with mismatched uncertainties via DOBC approach. ISA Trans 2011;50(3):389-96.

[11] Yang J, Li S, Sun C, Guo L. Nonlinear-disturbance-observer-based robust flight control for airbreathing hypersonic vehicles. IEEE Trans Aerosp Electron Syst 2013;49(2):1263-75

[12] Xue W, Bai W, Yang S, Song K, Huang Y, Xie H. ADRC with adaptive extended state observer and its application to air-fuel ratio control in gasoline engines. IEEE Trans Ind Electron 2015;62(9):5847-57.

[13] Chen WH, Yang J, Guo L, Li S. Disturbance-observer-based control and related methods. an overview. IEEE Trans Ind Electron 2016;63(2):1083-95.

[14] Li S, Yang J, Chen WH, Chen X. Generalized extended state observer based control for systems with mismatched uncertainties. IEEE Trans Ind Electron 2012;59(12):4792-802.

[15] Castillo A, García P, Sanz R, Albertos P. Enhanced extended state observerbased control for systems with mismatched uncertainties and disturbances. ISA Trans 2017;73:1-10.

[16] Zhao S, Gao Z. Modified active disturbance rejection control for time-delay systems. ISA Trans 2014:53(4):882-8.

[17] Zheng Q, Gao Z. Predictive active disturbance rejection control for processes with time delay. ISA Trans 2014;53(4):873-81.

[18] Sanz R, Garcia P, Albertos P. Enhanced disturbance rejection for a predictorbased control of LTI systems with input delay. Automatica 2016;72:205-8.

[19] Sanz R, Garcia P, Fridman E, Albertos P. Rejection of mismatched disturbances for systems with input delay via a predictive extended state observer. Int J Robust Nonlinear Control 2018;28(6):2457-67.

[20] Hao S, Liu T, Zhou B. Predictor-based output feedback control design for sampled systems with input delay subject to disturbance. IET Control Theory Appl 2017;11(18):3329-40.

[21] Hao S, Liu T, Wang QG. Enhanced active disturbance rejection control for timedelay systems. IFAC-PapersOnLine 2017;50(1):7541-6.

[22] Liu T, Hao S, Li D, Chen WH, Wang OG. Predictor-based disturbance rejection control for sampled systems with input delay. IEEE Trans Control Syst Technol 2017;(99):1-9.

[23] González A. Robust stabilization of linear discrete-time systems with timevarying input delay. Automatica 2013;49(9):2919-22.

[24] Gu K, Chen J, Kharitonov V. Stability of time-delay systems. Springer Science \& Business Media; 2003.

[25] Sanz R, Garcia P, Zhong Q, Albertos P. Predictor-based control of a class of time-delay systems and its application to quadrotors. IEEE Trans Ind Electron 2017;64(1):459-69. 